\title{
S-nitrosylation boon to blood transfusions
}

\section{By Kai-Jye Lou, Senior Writer}

Red blood cell transfusions are among the most commonly performed medical procedures, but the capacity of the transfused cells to transport oxygen is often reduced because of biochemical changes in the cells during storage. Now, Case Western Reserve University and Duke University Medical Center researchers have improved oxygen delivery capacity by re-nitrosylating hemoglobin prior to transfusion. ${ }^{1}$

The researchers are planning a series of clinical trials to determine whether the procedure can decrease the incidence of transfusionrelated adverse events compared with the current standard of care and potentially improve patient outcomes.

\section{"Most practitioners} consider fresh banked blood very efficacious, but the authors are arguing that since even the freshest blood from the blood bank has lost most of its S-nitrosohemoglobin, even these blood units could be made more effective by re-nitrosylation."

- John Roback, Emory University School of Medicine opposite of what is intended and actually decrease tissue oxygenation, possibly because of these storage-induced changes. ${ }^{3,4}$

Other studies have associated RBC transfusions with negative outcomes in some indications such as myocardial infarction (MI) and in the cardiac surgery setting, ${ }^{5-8}$ although it is unclear whether diminished oxygen delivery is the culprit.

In 2007, a group led by Jonathan Stamler reported that banked RBCs have a deficiency in $S$-nitrosohemoglobin levels. In a canine model, his team found that correcting the deficiency with a process called $S$-nitrosylation increased both the vasodilatory activity of RBCs and blood flow to tissues compared with what was seen in stored, untreated blood. ${ }^{9}$ Blood flow is a key determinant of oxygen delivery to tissues.

"The reality is that oxygen content in blood appears to be separate and distinct from its ability to deliver oxygen to organs and tissues," said Stamler, a professor of medicine and director of the Institute for Transformative Molecular Medicine at Case Western. "We have come to understand and are making the case that the delivery of oxygen to tissues by red blood cells is reflective of their ability to promote vasodilation and blood flow."

Now, Stamler's group has solidified the relationship between $S$-nitrosylation of hemoglobin in stored RBCs and the ability of those cells to deliver oxygen.

In mice, transfusion of re-nitrosylated, stored mouse RBCs maintained baseline muscle oxygenation levels, whereas transfusion of untreated, stored RBCs decreased muscle oxygenation levels.

In a rat model for hemorrhage, transfusion of re-nitrosylated, stored rat RBCs restored muscle oxygenation levels to baseline, whereas transfusion of untreated, stored RBCs did not.

In two sheep models for anemia, transfusion of re-nitrosylated, stored sheep RBCs increased muscle oxygenation and cardiovascular and renal function compared with transfusion of untreated, stored RBCs.

Results were published in the Proceedings of the National Academy of Sciences.

"Our work shows that re-nitrosylation has profound effects on the ability of stored red blood cells to improve blood flow, oxygen delivery to tissues and organs and organ function," said Stamler, corresponding author on the paper. "Our data suggest that it really is oxygen delivery that goes awry as stored red blood cells age. The findings also raise the idea that nitrosylated blood may be used therapeutically, in conditions such as myocardial infarction, to improve blood flow."

"The fact that they employed four different models is important as it decreases the possibility that the observed results were due to model-specific effects," said John Roback, an associate professor in the Department of Pathology and Laboratory Medicine and director of the Center for Transfusion and Cellular Therapy at the Emory University School of Medicine.

Roback noted that he also found the suggestion to apply renitrosylation to all banked blood provocative. "Most practitioners consider fresh banked blood very efficacious, but the authors are arguing that since even the freshest blood from the blood bank has lost most of its $S$-nitrosohemoglobin, even these blood units could be made more effective by re-nitrosylation," he told SciBX.

"Because banked red blood cells appear to become depleted of $S$-nitrosohemoglobin after just a few days in storage, the renitrosylation strategy will most likely need to be used in the hospital setting and applied just prior to transfusion," added Daniel KimShapiro, a professor in the Department of Physics and director of the Translational Science Center at Wake Forest University.

\section{Affecting adverse events and efficacy}

Stamler said his group is planning a clinical trial to obtain baseline measurements on how transfusions with stored RBCs change oxygen delivery and then determine how such changes correlate with transfusionrelated adverse events. He said his group has received a grant from the NIH to carry out the trial. He declined to disclose the grant amount. 


\section{ANALYSIS}

"Such information will be needed before we can properly assess the ability of re-nitrosylation therapy to improve oxygen delivery in transfusion recipients and whether use of re-nitrosylated red blood cells will have an impact on clinical outcomes," Stamler told SciBX.

Patients who receive RBCs can develop severe transfusion-related complications that lead to MI, organ injury and organ failure, ${ }^{9,10}$ though the incidences are estimated to be below $0.1 \%{ }^{11}$

Kim-Shapiro said it will be important to determine how renitrosylation affects the mechanical properties of RBCs and the chemical by-products that are generated from the re-nitrosylation procedure.

Other questions are how long the re-nitrosylation strategy takes, how durable it is and how long hemoglobin in treated RBCs stays nitrosylated, said Timothy McMahon, an associate professor of medicine at the Duke University School of Medicine. He was not involved in the current study.

Roback said cost considerations are especially significant in the blood transfusion setting, as it is the most common medical procedure performed at many hospitals and the number-one line item on hospital expense reports.

"It would be difficult at this time for blood banks to consider implementing such a technology, as they have been focusing on ways to decrease expenses," he told SciBX.

He noted that although the ethyl nitrite-based re-nitrosylation method used on sheep RBCs appears to be the most viable of the three methods to take into the clinical trials, it likely would add to the cost of a transfusion. Stamler's group re-nitrosylated mouse RBCs with purified nitric oxide (NO), rat RBCs with $S$-nitrosocysteine and sheep RBCs with ethyl nitrite.

Roback said that clinical data showing that re-nitrosylated RBCs improve patient outcomes over untreated RBCs are going to be needed before hospitals and blood banks consider whether to adopt the approach.

Duke and Case Western have multiple issued and filed patents covering re-nitrosylation of blood. Licensing details are available from the Case Western Reserve University Technology Transfer Office.

Lou, K.-J. SciBX 6(27); doi:10.1038/scibx.2013.673

Published online July 18, 2013

\section{REFERENCES}

1. Reynolds, J.D. et al. Proc. Natl. Acad. Sci. USA; published online June 24, 2013; doi:10.1073/pnas.1306489110

Contact: Jonathan S. Stamler, Case Western Reserve University, Cleveland, Ohio

e-mail: jonathan.stamler@case.edu

2. Gladwin, M.T. \& Kim-Shapiro, D.B. Curr. Opin. Hematol. 16, 515-523 (2009)

3. Hébert, P.C. \& Fergusson, D.A. JAMA 292, 1610-1612 (2004)

4. Tsai, A.G. et al. Transfusion 44, 1626-1634 (2004)

5. Sherwood, M.W. \& Rao, S.V. Nat. Rev. Cardiol. 10, 186-187 (2013)

6. Tinmouth, A. et al. Transfusion 46, 2014-2027 (2006)

7. Murphy, G.J. et al. Circulation 116, 2544-2552 (2007)

8. Netzer, G. et al. Chest 132, 1116-1123 (2007)

9. Reynolds, J.D. et al. Proc. Natl. Acad. Sci. USA 104, 17058-17062 (2007)

10. Malone, D.L. et al. J. Trauma 54, 898-905 (2003)

11. Hendrickson, J.E. \& Hillyer, C.D. Anesth. Analg. 108, 759-769 (2009)

COMPANIES AND INSTITUTIONS MENTIONED

Case Western Reserve University, Cleveland, Ohio Duke University Medical Center, Durham, N.C. Duke University School of Medicine, Durham, N.C. Emory University School of Medicine, Atlanta, Ga. National Institutes of Health, Bethesda, Md. Wake Forest University, Winston-Salem, N.C. 